\title{
Cryo-Electron microscopy for the study of self-assembled poly(ionic liquid) nanoparticles and protein supramolecular structures
}

\author{
Zdravko Kochovski $^{1}$ (D) Guosong Chen ${ }^{2}$ (D) Jiayin Yuan $^{3} \cdot{\text { Yan } \mathrm{Lu}^{1,4}}_{(\mathbb{D}}$ \\ Received: 16 January 2020 /Revised: 8 April 2020 / Accepted: 9 April 2020 / Published online: 23 May 2020 \\ (C) The Author(s) 2020
}

\begin{abstract}
Cryo-electron microscopy (cryo-EM) is a powerful structure determination technique that is well-suited to the study of protein and polymer self-assembly in solution. In contrast to conventional transmission electron microscopy (TEM) sample preparation, which often times involves drying and staining, the frozen-hydrated sample preparation allows the specimens to be kept and imaged in a state closest to their native one. Here, we give a short overview of the basic principles of Cryo-EM and review our results on applying it to the study of different protein and polymer self-assembled nanostructures. More specifically, we show how we have applied cryo-electron tomography (cryo-ET) to visualize the internal morphology of self-assembled poly(ionic liquid) nanoparticles and cryo-EM single particle analysis (SPA) to determine the three-dimensional (3D) structures of artificial protein microtubules.
\end{abstract}

Keywords Self-assembly $\cdot$ Poly(ionic liquid) nanoparticles $\cdot$ Protein self-assembly $\cdot$ Cryo-electron microscopy $\cdot$ Single particle analysis · Cryo-electron tomography

\section{Introduction}

Self-assembly of proteins plays a key role in many biological processes and has attracted substantial interest in recent years due to various potential applications in diagnostics and biosensing [1] or the design of biocompatible and biodegradable materials [2]. The design and construction of artificial protein nanostructures through strategies for self-assembly offer a unique way of understanding the basic principles for the creation of diverse functional materials with advanced properties. For similar reasons, polymer self-assembly has also become a topic of increasing

Yan Lu

yan.lu@helmholtz-berlin.de

1 Institute of Electrochemical Energy Storage, Helmholtz-Zentrum Berlin für Materialien und Energie, 14109 Berlin, Germany

2 The State Key Laboratory of Molecular Engineering of Polymers and Department of Macromolecular Science, Fudan University, Shanghai 200438, China

3 Department of Materials and Environmental Chemistry, Stockholm University, SE-106 91 Stockholm, Sweden

4 Institute of Chemistry, University of Potsdam, 14476 Potsdam, Germany interest in recent years [3]. While TEM has established itself as the standard technique of choice for the structural and morphological analysis of self-assembled nanostructures, conventional TEM sample preparation often involves drying and staining steps which in many cases can affect the structure and morphology of the sample. In the case of cryo-EM, the aqueous samples are preserved in a frozen-hydrated state, closest to their native one, through vitrification and studied in the TEM at cryogenic temperatures. Cryo-EM is nowadays a powerful method for structure determination that has also been recently awarded with the 2017 Nobel Prize in Chemistry. The two main cryo-EM three-dimensional (3D) structure determination techniques are single-particle analysis (SPA) and cryo-electron tomography (cryo-ET). Here, we outline the basic principles of both techniques, including sample preparation, and give examples of how we have employed them in the study of protein and polymer self-assembled nanostructures.

\section{Sample preparation}

Negative staining is a commonly used conventional sample preparation method for soft matter TEM samples and basically consists of embedding the sample in a film of heavy metal salt 
[4]. In one way, it serves as a means to overcome the low contrast of organic samples made out of light elements, since the film scatters electrons more strongly than the embedded material. It also provides some degree of structural preservation when the samples are exposed to high-vacuum in the TEM column and to a certain degree reduces the radiation damage caused by high-energy electrons hitting the sample. However, as the stain cannot penetrate small crevices in the sample, most of the time, only an envelope of the embedded molecules could be imaged with a resolution that is limited by the grain size of the stain [5]. Additionally, the sample's structural features may be affected by drying and staining artifacts. Regardless of its drawbacks, negative staining can play an important role in the initial sample quality evaluation and optimization for cryo-EM [6].

In contrast to conventional TEM sample preparation techniques, samples for cryo-EM are preserved in a frozen hydrated, close to physiological, state through rapid cooling (vitrification) [7]. This is typically done by plunge freezing of the sample into a cryogen with good heat-transfer properties, such as liquid nitrogen-cooled liquid ethane. Normally, several microliters of the sample are applied to a holey carbon-coated TEM grid, the solution is then blotted away with filter paper, leaving a meniscus of water in the holes of the film, and the grid is then immediately plunged into the cryogen. The samples are cooled so rapidly (cooling speeds in the order of $10^{5}{ }^{\circ} \mathrm{Cs}^{-1}$ are normally reached) that ice crystals do not have time to form, producing the so-called vitreous ice that is amorphous and similar to a liquid with a very high viscosity. Cryo-EM samples must be kept at all times at temperatures below $-135^{\circ} \mathrm{C}$ in order to avoid devitrification and the formation of ice crystals.

\section{Cryo-EM SPA}

\section{Basic principles of SPA}

Single-particle cryo-EM is based on combining TEM images of individual isolated macromolecules at different orientations, i.e., single particles, into a 3D reconstruction. Normally thousands of images of individual complexes need to be collected, aligned, and averaged before a high-resolution 3D reconstruction can be produced [8]. The principle of obtaining a reconstruction of a 3D object from a set of two-dimensional (2D) projections of the object at different views relies on the socalled "projection-slice theorem" [9]. It states that the Fourier transform of a $2 \mathrm{D}$ projection from a $3 \mathrm{D}$ object corresponds to a central slice in the 3D Fourier transform of that object, and basically means that by obtaining enough $2 \mathrm{D}$ projections of the object at different orientations, its entire Fourier space can be sampled and the object can be then reconstructed by Fourier inversion. For this to work, however, the relative orientations of the $2 \mathrm{D}$ projections have to be determined, which is normally done by an iterative algorithm called "projection matching" [ 9 , 10]. In this approach, one starts with an initial 3D reference structure from which a set of projections (also known as "reprojections") in all different directions are generated and then computationally compared with the experimental singleparticle images in order to determine their relative orientations. Once this is done, a new 3D reference can be calculated from the experimental images and the whole process proceeds iteratively, usually until no significant improvement in the resolution of the newly obtained 3D reconstruction is achieved upon further iterations. Given that the experimental data is of high quality, with a good angular coverage and no preferred particle orientations (known as "orientation bias"), this approach normally converges to the correct structure even with an initial reference that does not closely resemble the true structure, such as a low-resolution representation [9] or even random densities [10]. If this is not the case, the result could be a so-called model bias effect where the projection matching algorithm is stuck in a local minimum with the final structure being influenced by the choice of the initial reference. One way to avoid or at least identify model bias is to use an ab initio-derived model from the experimental data. Two common approaches in generating such models are the random conical tilt [11] and the "common lines" [12]. The latter approach can determine relative orientations between pairs of projections by making use of the fact that each pair of 2D projections of an object shares a common line in the 3D Fourier transform of that object (resulting from the projection-slice theorem discussed above). The random conical tilt approach, on the other hand, makes use of preferred molecule orientations and employs a single calibrated tilt of the TEM sample stage to generate an unbiased 3D reference.

One of the major limiting factors in cryo-EM of soft matter is radiation damage, caused by the interaction of the highenergy electrons with the sample. In order to minimize radiation damage, the electron dose in cryo-EM SPA is normally limited to $20 \mathrm{e}^{-} / \AA^{2}$, which results in a low signal-to-noise ratio (SNR) of the images. Averaging over tens of thousands of images of the molecule of interest is normally needed in order to gain signal. Obtaining a reliable $3 \mathrm{D}$ reconstruction by cryoEM SPA requires a sample where the particles are in their native state, embedded in a thin layer of ice and ideally assume random orientations. Producing such samples is one of the major bottlenecks associated with this technique. Orientation bias is less of a problem in the cryo-EM SPA for helical assemblies as the relative orientations of the molecule can be inferred from the helical symmetry [13].

\section{Recent technological developments}

Just several years back, the typical resolution achievable by cryo-EM SPA was rather poor (worse than $10 \AA$ ), which earned the method the nickname "blob-ology". Due to a number of recent technological advances in both hardware and 
software, this technique is now capable of determining the atomic structures of challenging macromolecules and rivals X-ray crystallography in terms of resolution. Probably the most important innovation in terms of hardware that has led to this "resolution revolution" [14] is the development and commercialization of direct electron detectors that can record images at high-speed and with greatly improved SNR compared to their predecessors such as scintillator-coupled CCD cameras or film [15]. The high speed of data acquisition of this new generation of detectors (typically hundreds of frames per second) combined with their improved SNR has allowed for the fractionation of the total electron dose of a single image exposure into multiple sub-frames, which has enabled the compensation for specimen motion, drift, and radiation damage by carrying out a computational frame alignment post acquisition [16]. Almost at the same time, novel and powerful maximum likelihood image processing algorithms have become available that could handle the high volumes and complexity of data coming from these new detectors in a reliable and objective manner and with a limited amount of user intervention [17]. Another important recent innovation that is worth mentioning is the Volta potential phase plate for infocus phase contrast TEM imaging [18]. This new type of TEM phase plate has solved some of the problems typically associated with its predecessors (e.g., Zernike-type phase plates [19]), such as short life span and fringing artifacts, and has made it possible to successfully apply cryo-EM SPA to difficult samples, such as small proteins and flexible complexes [20]. Although cryo-EM SPA is traditionally associated with the analysis of biological systems, recently it has also been successfully applied to the structure determination of synthetic inorganic nanomaterials with cage-like shapes [21]. In this study, isolated chemically and structurally homogeneous silica cages ("silicages") with a dodecahedral structure and a size of less than $10 \mathrm{~nm}$ have been generated and analyzed by cryo-EM SPA with the reconstruction reaching a resolution of around $2 \mathrm{~nm}$ (Fig. 1). Reaching a close to atomic resolution in that case has been most probably hampered by the amorphous nature of silica at the atomic level in these cages [21]. Nevertheless, cryo-EM will likely be adopted for the analysis of similar systems in the future as an understanding of the 3D structure of synthetic nanoparticles becomes more and more important.

\section{Cryo-ET}

\section{Basic principles of Cryo-ET}

Similar to SPA, cryo-ET enables the three-dimensional visualization of frozen-hydrated samples. However, in tomography the $2 \mathrm{D}$ projections required for the $3 \mathrm{D}$ reconstruction are obtained from a series of images of a single specimen area (tilt series) acquired by physically tilting the computer-controlled specimen stage to different angular orientations [22, 23]. The collected micrographs can then be computationally merged into a 3D reconstruction (tomogram) (Fig. 2). Due to physical limitations imposed by the stage and sample holder design, the tilting range is typically limited to $\pm 70^{\circ}$. This results in a wedge-shaped region of missing information in Fourier space and causes non-isotropic resolution in the reconstruction with structural features showing an elongation in the beam direction $[24,25]$. Tilt acquisition schemes that can minimize these unwanted effects, such as dual-axis tomography [26] where one acquires a pair of tilt series around two orthogonal axes exist but are still hardly used, mostly due to inherent technical difficulties. Weighted back-projection [27] is one of the most widely used 3D reconstruction methods in cryo-ET. It basically backprojects the 2D projections to 3D space and compensates for the uneven distribution of information in the Fourier space that this process causes. Iterative reconstruction algorithms such as the simultaneous iterative reconstruction technique (SIRT) [28] and the algebraic reconstruction technique (ART) [29] are other alternatives that work by minimizing the difference between the experimental 2D projections and the reprojections of the 3D image. As cryo-ET does not rely on the presence of multiple copies of the structure of interest, it is well-suited for the analysis of complex and pleomorphic structures. Due to the sensitivity of cryo-preserved soft matter samples to the electron beam, tilt series acquisition has to be done under low-dose conditions and the total electron dose distributed among the images, which generally results in a low SNR in the $2 \mathrm{D}$ projections and $3 \mathrm{D}$ reconstruction. Although the nominal resolution in cryo-ET depends on a number of technical parameters, such as pixel size at the specimen level, angular range, and increment, it is the low SNR that generally limits the usable resolution. Subtomogram averaging is a technique that can be utilized to improve the resolution in cryo-ET, given that identical copies of structures of interest (e.g., macromolecular complexes or virus particles) are present in a tomogram. Subtomograms containing identical copies of the structure of interest are selected, aligned, and averaged in order to improve the SNR and resolution, and also minimize the effects produced by the missing wedge. One additional limitation of the technique is the sample thickness, and for samples thicker than about $500 \mathrm{~nm}$, some type of thinning under cryogenic conditions is required. Currently, the state-of-the-art method for achieving cryogenic-focused ion beam milling (cryo-FIB) where a focused beam of gallium ions is used to ablate material at selected regions on the sample at a precision of several tens of nanometers [30].

\section{Segmentation}

Often, analyzing tomographic data requires the separation and extraction of the features of interest from the noisy 


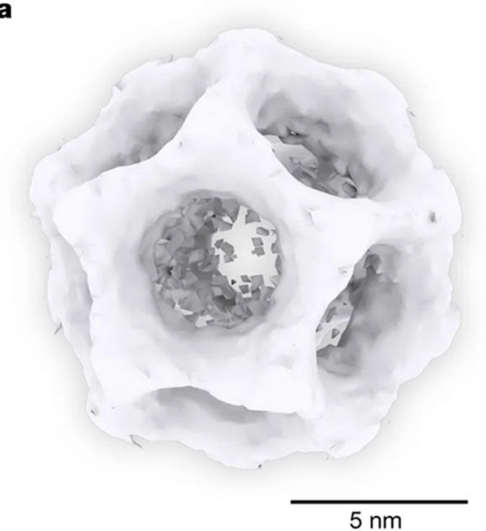

C 3D model
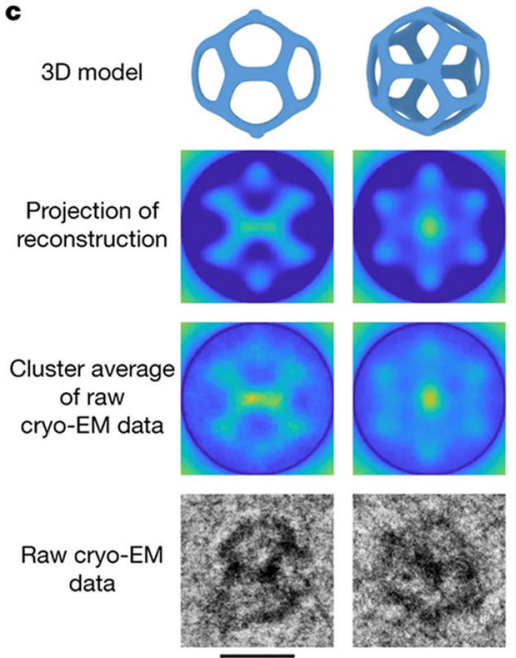

Fig. 1 Single-particle reconstruction of a dodecahedral silicage. Reconstruction of a dodecahedral silicage (a) and its three most unique projections along the twofold, threefold, and fivefold symmetry axes (b). The average dimensions of silicages in $\mathbf{b}$ were estimated from the reconstructed dodecahedral silicage. c Representative comparison of nine unique projections from the reconstruction and cryo-EM cluster
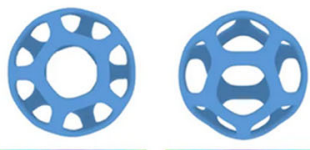
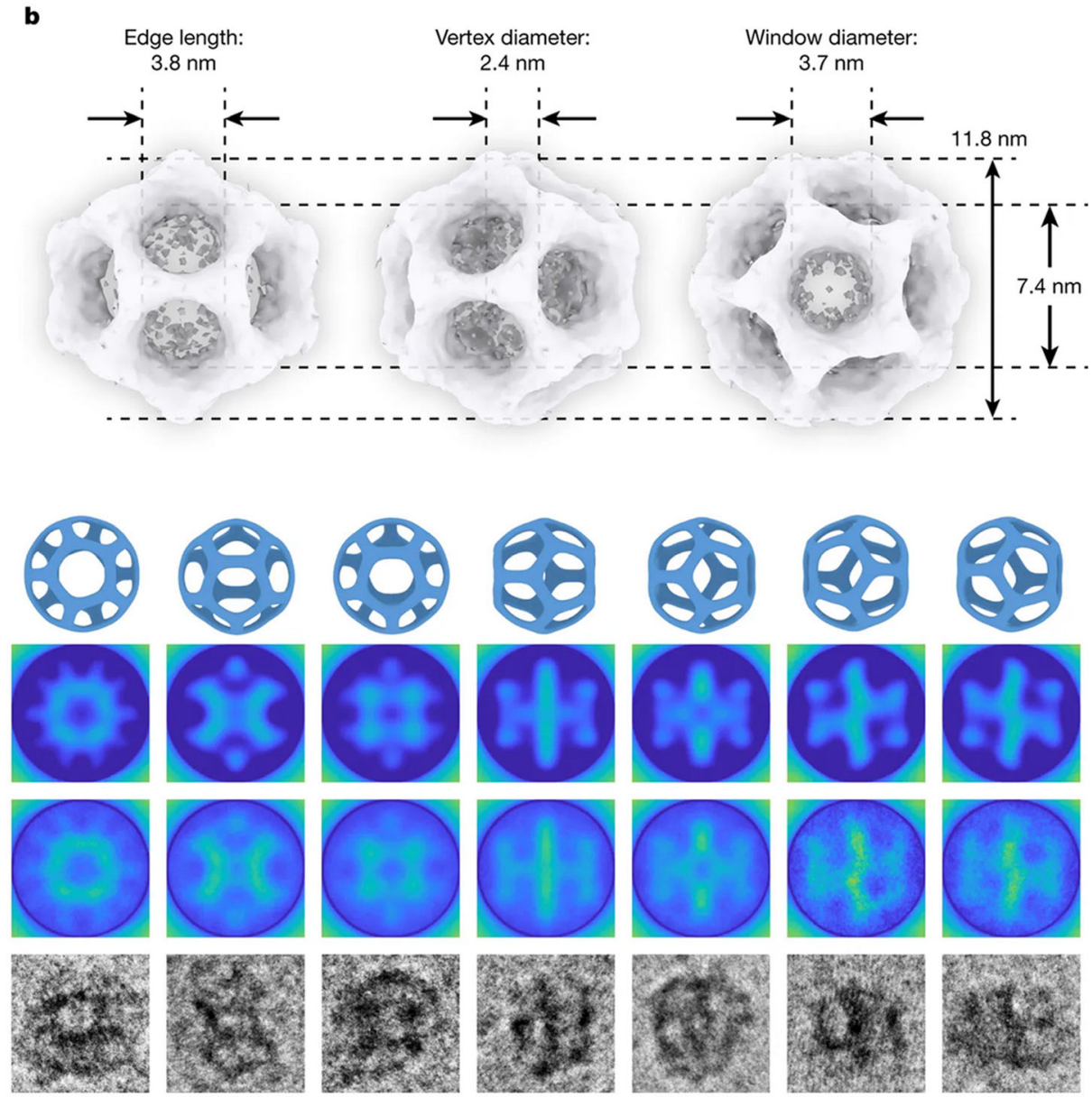

averages with projections of a 3D dodecahedral cage model. Corresponding single cryo-EM images are shown at the bottom, highlighting the difference between the raw data and the reconstruction. The scale bar in c represents $10 \mathrm{~nm}$. Reprinted by permission from Macmillan Publishers Ltd., Nature [21], copyright 2018
Fig. 2 General electron

tomography workflow. a The

sample is tilted inside the TEM

column and a set of images called a tilt series is collected. $\mathbf{b}$ The tilt series is aligned and reconstructed to a $3 \mathrm{D}$ volume or tomogram. $\mathbf{c}$ The features of interest in the tomogram can be separated by segmentation. Here the segmentation shows a gold nanoparticle (yellow) covered by a silica shell (blue)

a

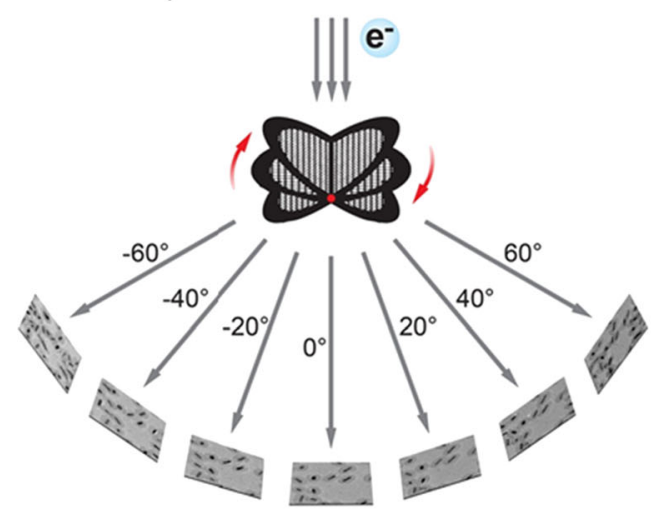

Tilt series acquisition
C

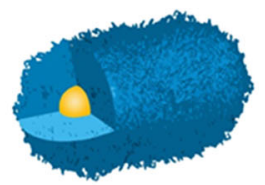

Segmentation

b

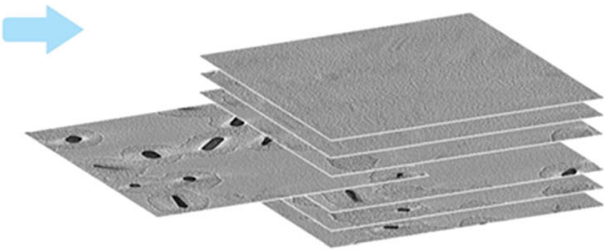

3D reconstruction 
background, a process commonly referred to as segmentation (Fig. 2). Manual segmentation has long been the most common type of image segmentation used for the feature extraction in cryo-ET, owing to the inherently noisy nature of the data. This approach is however prone to user bias and can be exceptionally time-consuming. Automated segmentation approaches capable of generating more objective and reproducible results are increasingly being developed [31-33], which is in part due to the increase in data quality that has been made possible by some of the technological developments discussed in the previous section.

\section{Polymer self-assembly: Poly(ionic liquid) nanoparticles}

Poly(ionic liquid)s (PILs) are a class of innovative ionic macromolecules that combine some of the unique characteristics of ionic liquids with the more familiar properties of polymers, such as mechanical stability and durability as well as rich mesostructures by self-assembly. This combined property profile makes them suitable for a number of applications such as $\mathrm{CO}_{2}$ sorbents, carbon precursors, dispersants, and porous materials, to name a few $[34,35]$. PIL self-assembly has raised considerable interest in recent years due to their potential applications for the bottom-up assembly of functional nanostructured materials [36, 37]. Among these PIL self-assemblies, the ones that catch the most interest are a series of well-defined homopolymer nanoparticles prepared via a one-pot self-stabilizing dispersion polymerization of 1-vinyl-3alkylimidazolium bromide and 1-vinyl-4-alkyl-1,2,4triazolium bromide. These IL monomers feature an ionic imidazolium or triazolium head with a long hydrophobic alkyl chain from dodecyl C12 to hexadecyl C16. It is found that the inner structure of the PIL nanoparticles is sensitive to the alkyl chain length, and the type of the anions ( $\left(\Gamma\right.$ or $\left.\mathrm{Br}^{-}\right)$.

By varying the length of the alkyl tails associated with the imidazolium or triazolium cations, we were able to tune the interior and outer shapes of the nanoparticles with sub-5 nm nanodomain spacing. This change can be visualized and investigated by cryo-EM and cryo-ET in detail. A morphological evolution of particle shape and interior occurs at this extremely small size scale that is uncommon for colloids derived from homopolymers [38]. Here, the PIL nanoparticles poly(TILM-CnX) produced from 1-vinyl-4-alkyl-1,2,4triazolium bromide are taken as an example, where $n$ denotes the number of carbons in the alkyl chain and $X$ denotes the anions.

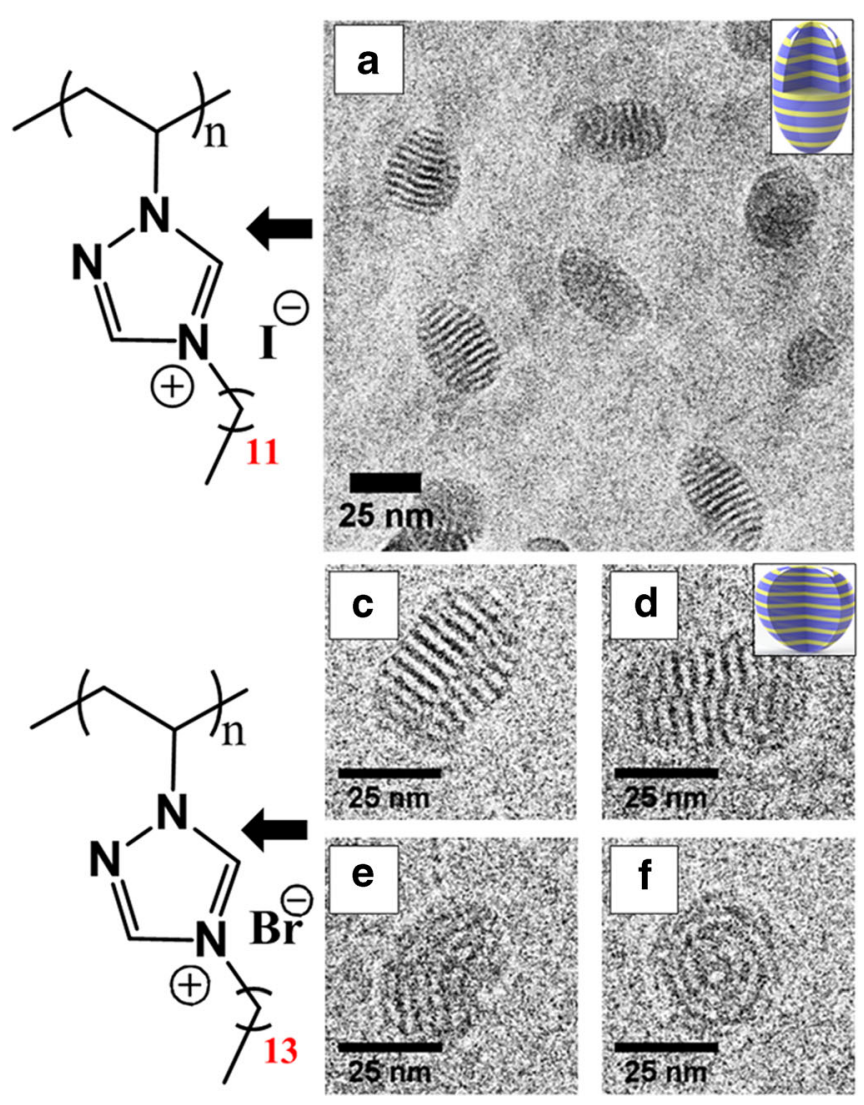

Fig. 3 Representative cryo-EM images of PIL nanoparticles in aqueous solution. a poly(TILM-C12I), b poly(TILM-C12Br), c-f poly(TILM$\mathrm{C} 14 \mathrm{Br})$, and $\mathbf{g}$ poly(TILM-C16Br). The chemical structures correspond

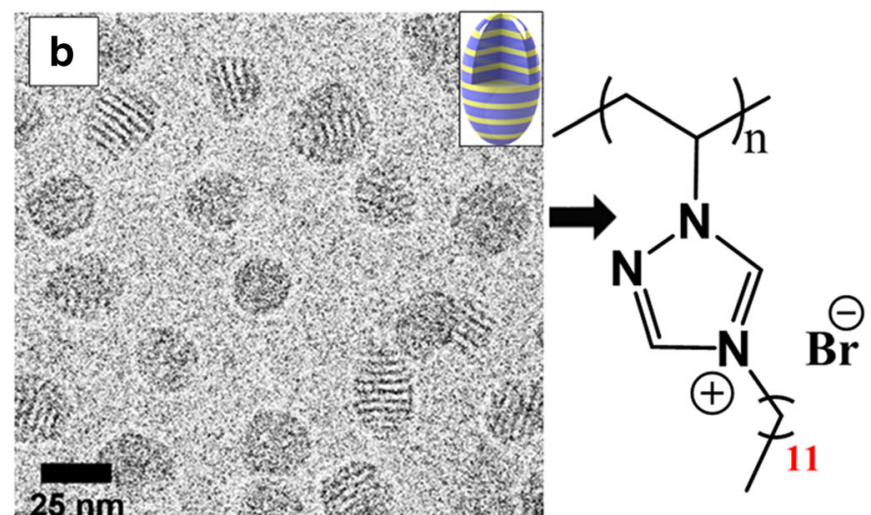

$25 \mathrm{~nm}$. . . . n

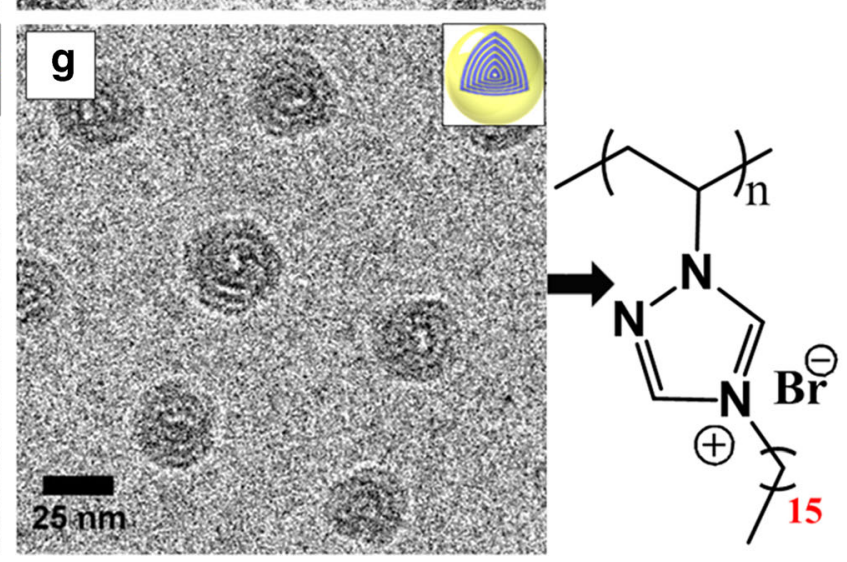

to individual PIL nanoparticles. Figure adapted with permission from ACS [38]. Copyright 2016 American Chemical Society 
Cryo-EM micrographs in Fig. 3 visualized the morphologies of poly(TILM-CnX) nanoparticles with varied alkyl tail length, which already revealed the sensitivity of their shape and interior toward subtle variation in their chemical structure. For example, the poly(1-vinyl-4-dodecyl-1,2,4-triazolium iodide) nanoparticles poly(TILM-C12I) present a unique wasplike morphology with parallel lamellae (Fig. 3a). The light and dark lamellae stem from the hydrophobic dodecyl chain and the charged backbones, respectively. The contrast in these two domains originates from a higher electron density of the iodide atoms compared with that of the carbon and hydrogen atoms in the dodecyl chain. It should be noted that though the wasp-like morphology has been reported previously by block copolymers, the size of the particles are usually over $100 \mathrm{~nm}$ and the phase-separated domains are above $10 \mathrm{~nm}$. Here in the PIL nanoparticles, the domain size decreases significantly to below $5 \mathrm{~nm}$ and remains visible in TEM under cryogenic condition. When the anion is exchanged from iodide to bromide, the nanoparticles poly(TILM-C12Br) presents a similar wasp-like morphology to the poly(TILM-C12I) one, indicating that the subtle size shrinkage from iodide to bromide does not trigger a visible variation of the PIL nanoparticle morphology.

However, when extending the alkyl chain length from dodecyl C12 to hexadecyl C16, a centric multilamellar onionlike morphology was evolved instead (Fig. 3g). A close view of Fig. $3 \mathrm{~g}$ further reveals the presence of shape edges and facets on the onion-like morphology. Via differential scanning calorimetry (DSC) measurements of dried PIL samples, we found that at the sample preparation temperature $\left(4^{\circ} \mathrm{C}\right)$, only the alkyl chains in PILM-C16Br are dominantly in a crystalline state, which is responsible for the anisotropic edges and facets in PILM-C16Br. When the alkyl chain is at the tetradecyl length scale between the dodecyl and hexadecyl, a transition morphology combining both deformed wasp-like and onion-like structures was identified (Fig. 3c-f). Such sensitivity toward chemical structure derives from the phase separation at the extremely small size scale, here sub- $5 \mathrm{~nm}$, where the interfacial effect is amplified by the small nanosize and thus can drastically change its morphology to minimize
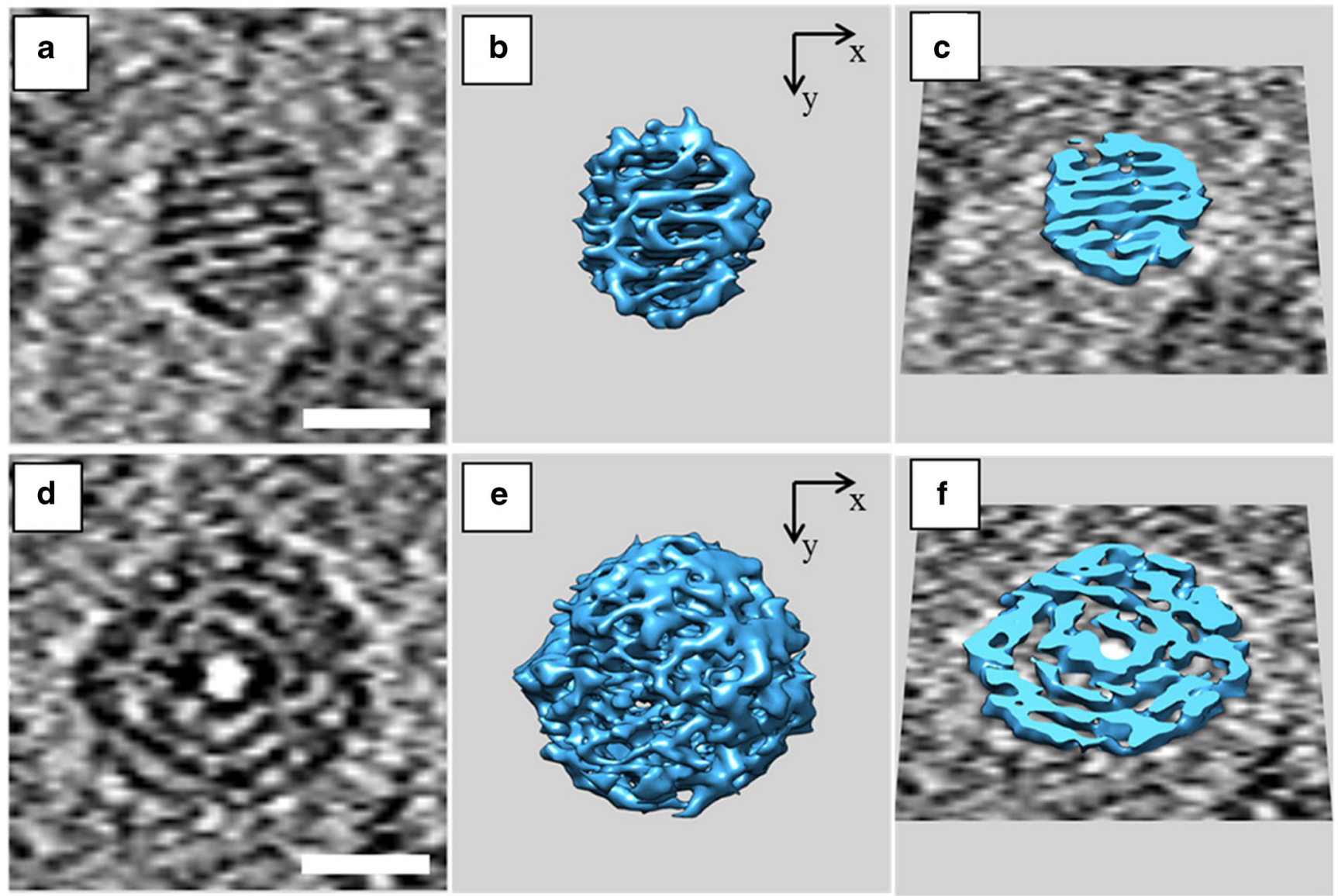

Fig. 4 Representative cryo-ET of PIL nanoparticles. a, d Central tomographic slices along the $Z$ axis (normal to the paper surface) of the tomographic volumes of individual poly(TILM-C12Br) and poly(TILM$\mathrm{C} 16 \mathrm{Br}$ ) particles, respectively (scale bar $25 \mathrm{~nm}$ ). b, e 3D volume renderings of the particles in a and d, respectively. c, f Same 3D volume renderings as in $\mathrm{b}$ and $\mathrm{e}$, but digitally clipped along the $Z$ axis around their central regions and shown together with the tomographic slices from a and $\mathrm{d}$. The $3 \mathrm{D}$ images in $\mathrm{c}$ and f have been slightly tilted around the $X$ axis in order to get a better representation of the internal features of the structures. Figure adapted with permission from ACS [38]. Copyright 2016 American Chemical Society 
Fig. 5 a and $\mathbf{b}$ Protein microtubes visualized by cryo-EM at different magnifications. c EM micrograph of negatively stained protein microtubes. Insets, enlarged versions of marked areas; scale bar, $10 \mathrm{~nm}$. d Slice $(0.8 \mathrm{~nm}$ thick, $x y$ ) through the 3D tomographic reconstruction of negatively stained protein microtubes. Figure adapted with permission from ACS [39]. Copyright 2016 American Chemical Society
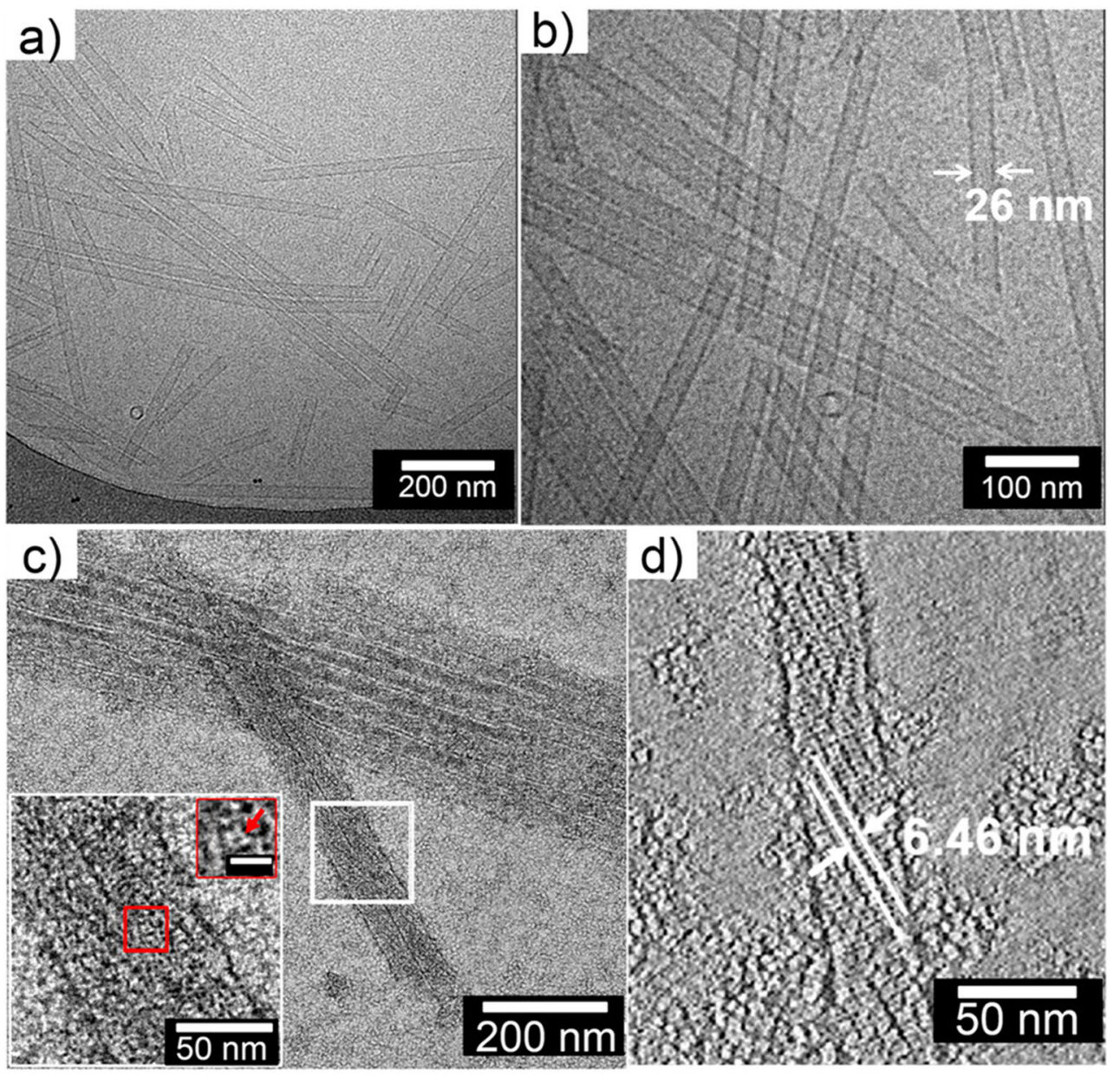

c)

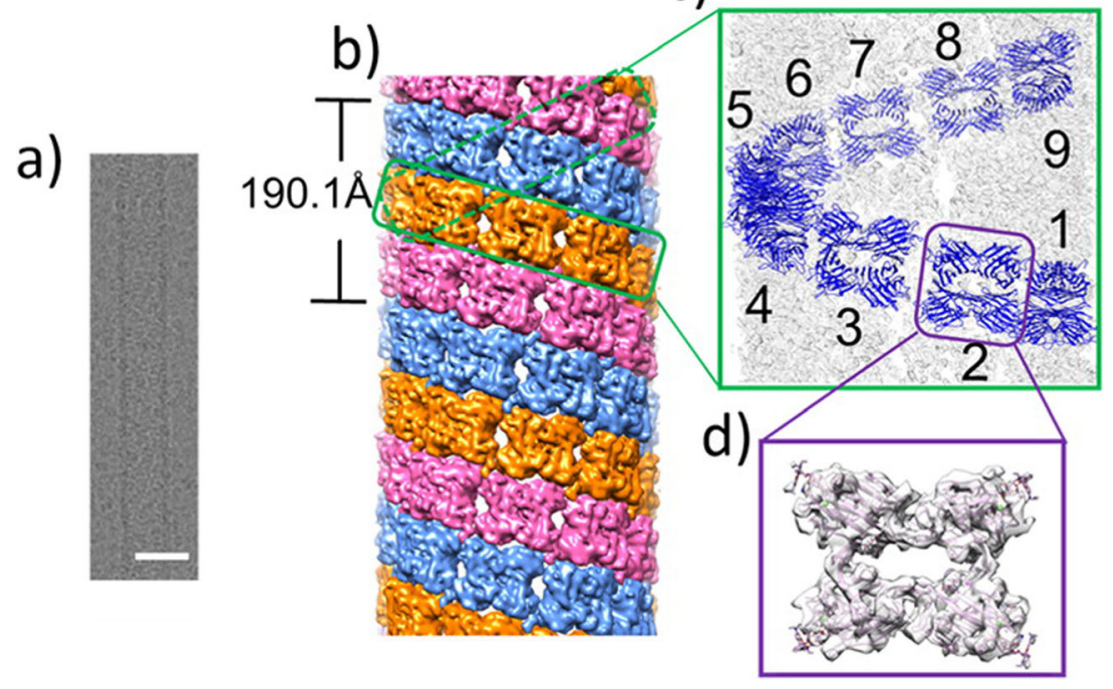

e)

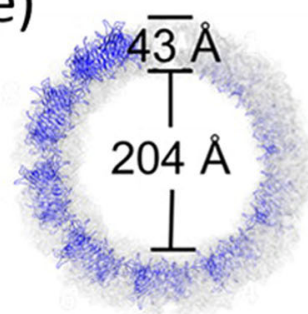

f)

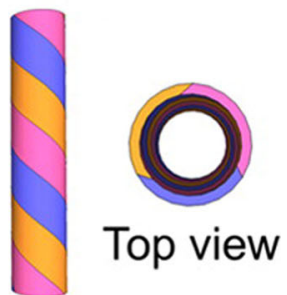

\section{Side view}

Fig. 6 Structural model of SBA microtube. a Single SBA microtube visualized by cryo-EM (Scale bar $25 \mathrm{~nm}$ ). b Electron density map of SBA microtube after $3 \mathrm{D}$ reconstruction. Three neighboring protofilaments are respectively colored in red, yellow, and blue. The length of each turn (periodicity) is $\sim 19 \mathrm{~nm}$. $\mathbf{c}$ Each turn is composed of nine identical tetrameric SBA molecules colored in blue. d Tetrameric SBA model fitted in the electron density map. e Top view of microtube model. The thickness of the tube wall is $\sim 4 \mathrm{~nm}$. The inner diameter of the microtube is $\sim 20 \mathrm{~nm}$. Each SBA tetramer in one periodic helical structure is shown in blue in c and e. f Cartoon model of the microtube. The three protofilaments are colored in red, yellow, and blue, respectively. Figure adapted with permission from ACS [39]. Copyright 2016 American Chemical Society 
Fig. 7 a and $\mathbf{b} 2 \mathrm{D}$ protein lattices visualized by cryo-EM at different magnifications, inset, the corresponding fast Fourier transform of selected area. $\mathbf{c}$ and $\mathbf{d} 1 \mathrm{D}$ protein filaments visualized by cryo$\mathrm{EM}$ at different magnifications. Figure adapted from [40] with permission from Wiley-VCH Verlag GmbH \& Co.
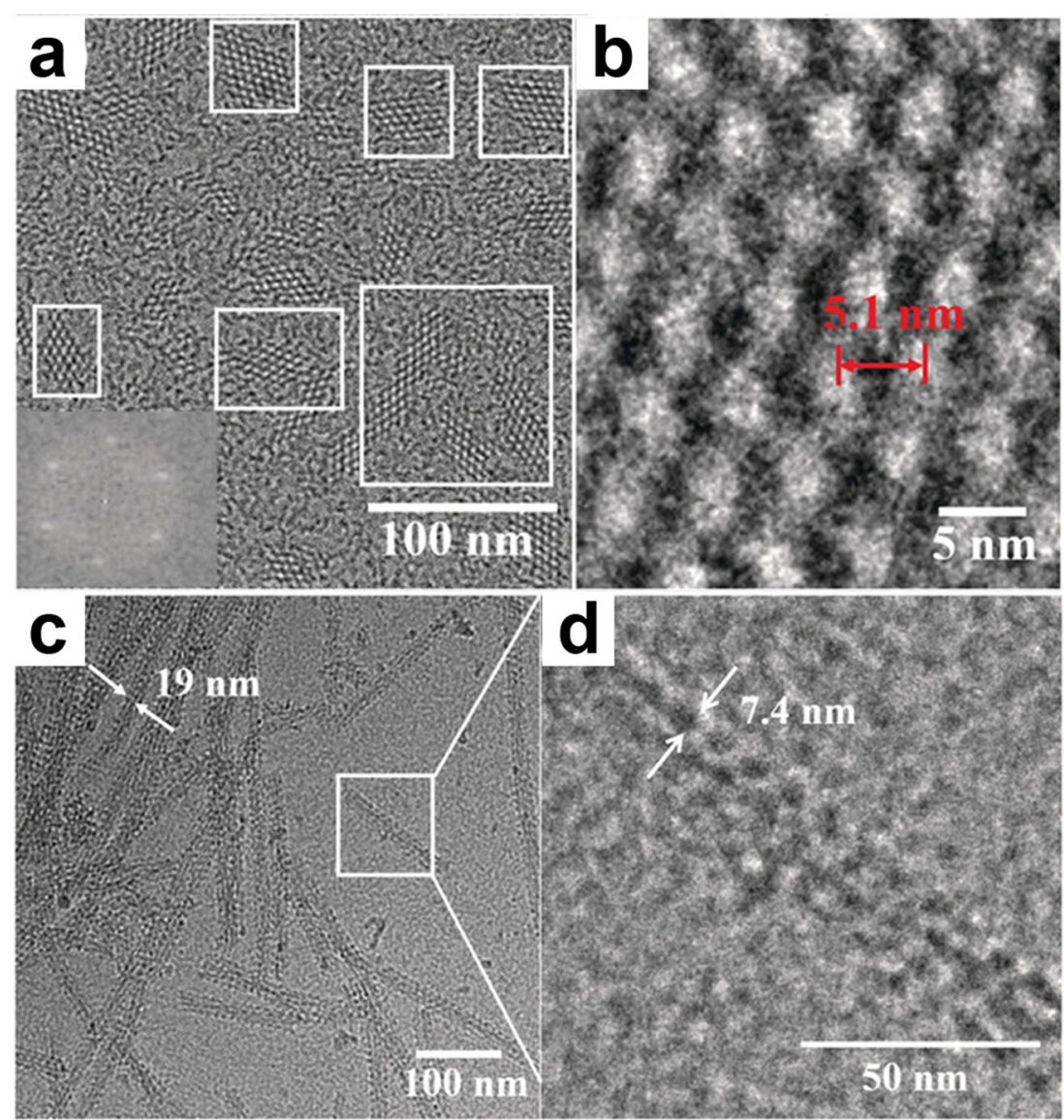

surface energy, even when the hydrophobicity and volume of the alkyl chain domain changes slightly by one or two more$\mathrm{CH}_{2}-$ units.

Using cryo-ET, we were able to shed more light into the internal morphology of these particles (Fig. 4), which is otherwise only seen via a 2D-projected image, as shown in Fig. 3. 3D renderings of "wasp-like"and"onion-like" particles were obtained by image segmentation of the tomographic volumes (Fig. 4b, e). For the poly(1-vinyl-4-hexadecyl-1,2,4triazolium bromide) type of particles (poly(TILM-C16Br)), we could reveal a $3 \mathrm{D}$ arrangement that approaches to the true inner structure of these nanoparticles. This $3 \mathrm{D}$ arrangement is unexpectedly different from the onion-like morphology suggested by the 2D cryo-EM images alone in Fig. 3g. What initially appeared to be an "onion-like" configuration turned out to be a "spaghetti ball" configuration (Fig. 4d, e, and f), where the hydrophilic shells (in dark) in the onion-like structure is perforated by the hydrophobic alkyl chains (in light). That is, the $2 \mathrm{D}$ projection can detect the alternating hydrophobic shell (built up from the alkyl chain) and the hydrophilic shell (built up from the ionic backbone) in an onion-like morphology, while 3D electron tomography further found that the hydrophilic shell is not fully closed but perforated by the hydrophobic alkyl chains. The perforation of the alkyl chains through the hydrophilic shell is driven by the large size of the alkyl chains, which are too big to be exclusively confined within the thin hydrophobic shell. In this regard, the cryo-EM $3 \mathrm{D}$ tomography is a really powerful characterization method that can better and more deeply approach the intrinsic inner structure of polymer nanoparticles. It should be noted that the sub-5 nm phase separation, previously seldom observed, now in poly(ionic liquid) nanoparticles is very possibly due to the strong hydrophilicy-hydrophobicity contrast between the long alkyl chain and the imidazolium bromide ionic pair, which stabilize the ultrasmall scale phase separation.

\section{Protein self-assemblies}

\section{Protein-microtubule-like structures}

Protein microtubules are remarkable self-assembled structures that provide shape and structure to eukaryotic cells. Designing artificial protein microtubules with precise structure and controllable self-assembly behavior has remained a challenge for a long time. We designed and achieved self-assembled protein-microtubule-like structures from tetrameric soybean agglutinin (SBA) without any chemical or biological 
modification using dual supramolecular interactions from a series of well-designed ligands, i.e., protein-sugar interaction and $\pi-\pi$ stacking [39]. Cryo-EM micrographs of the assemblies showed highly homogeneous microtubular structures with a micrometer-scale length and a width of around $26 \mathrm{~nm}$ (Fig. 5a, b). We additionally confirmed the tubular structure by cryo-ET. The diameter of the protein microtubule in micrographs of negatively stained samples was measured at around $36 \pm 2 \mathrm{~nm}$, which was attributed to the dehydration and collapse of the hollow structure (Fig. 5c). While this collapsed state was also confirmed by negative stain tomography, the protofilament periodicity and helical parameters necessary for the single particle reconstruction later on could still be determined from the tomogram of the collapsed structures (Fig. 5d). This is an example of how negative staining can play a key role in the evaluation of cryo-EM samples.

Using cryo-EM SPA we determined the structure of the artificial microtubule to a resolution of $7.9 \AA$ (Fig. 6). The $3 \mathrm{D}$ reconstruction revealed how the SBA tetramers selfassemble into a microtube featuring a twisted helical structure and also showed their detailed packing pattern and the location of ligands. The reconstructed map revealed three smooth protofilaments winding around a hollow core in a left-handed fashion (Fig. 6b, marked as red, yellow, and blue). Each protofilament exists as a periodic helical structure with a period of $\sim 19 \mathrm{~nm}$, while each of the periodic structures consists of nine tetrameric SBAs (Fig. 6c).

\section{D protein filaments and 2D protein lattices}

Protein structures with different dimensions are of great interest for biologists due to their great importance and abundance in living organisms. Whereas the main approaches to construct different protein structures are still engineering intensive and highly dependent on the accuracy of the protein design. We designed and targeted 1D protein filaments and 2D protein lattices from tetrameric LecA with a cuboid shape based on finely tuning the length of the linker moiety of inducing ligand [40]. When the linker length is relatively short or long, cryoEM micrographs showed highly ordered 2D protein lattices with a visible protein molecule (Fig. 7a, b). Notably, while the linker length is moderate, well-defined 1D protein filaments with a diameter of $7.4 \mathrm{~nm}$ was observed by cryo-EM (Fig. 7c, d).

\section{Conclusions}

In this work, we have given a brief overview of the two main cryo-EM three-dimensional (3D) structure determination techniques: single particle analysis (SPA) and cryo-electron tomography (cryo-ET). We have shown examples from our own work on how we have successfully applied both techniques to determine the 3D strictures of self-assembled poly(ionic liquid) nanoparticles and artificial protein microtubules. Furthermore, we have shown that cryo-EM 2D micrographs alone can be used to visualize different morphologies of self-assembled protein structures, such as 1D protein filaments and 2D protein lattices. Inspired by its success in the field of structural biology, we expect that cryo-EM will play an increasingly important role in the study of synthetic selfassembled systems.

Acknowledgments The authors also thank the Joint Lab for Structural Research at the Integrative Research Institute for the Sciences (IRIS Adlershof).

Author contributions The manuscript was written through contributions of all authors. All authors have given approval to the final version of the manuscript.

Funding information Open Access funding provided by Projekt DEAL. Y. L. would like to thank the Deutsche Forschungsgemeinschaft (DFG, German Research Foundation) — grant no. LU1423/1-1 — for the financial support.

\section{Compliance with ethical standards}

Conflict of interest The authors declare that they have no conflict of interest.

Open Access This article is licensed under a Creative Commons Attribution 4.0 International License, which permits use, sharing, adaptation, distribution and reproduction in any medium or format, as long as you give appropriate credit to the original author(s) and the source, provide a link to the Creative Commons licence, and indicate if changes were made. The images or other third party material in this article are included in the article's Creative Commons licence, unless indicated otherwise in a credit line to the material. If material is not included in the article's Creative Commons licence and your intended use is not permitted by statutory regulation or exceeds the permitted use, you will need to obtain permission directly from the copyright holder. To view a copy of this licence, visit http://creativecommons.org/licenses/by/4.0/.

\section{References}

1. Busseron E, Ruff Y, Moulin E, Giuseppone N (2013) Supramolecular self-assemblies as functional nanomaterials. Nanoscale 5:7098-7140. https://doi.org/10.1039/C3NR02176A

2. Lutolf M, Hubbell J (2005) Synthetic biomaterials as instructive extracellular microenvironments for morphogenesis in tissue engineering. Nat Biotechnol 23:47-55. https://doi.org/10.1038/nbt1055

3. Jin HB, Huang W, Zhu XY, Zhou YF, Yan DY (2012) Biocompatible or biodegradable hyperbranched polymers: from self-assembly to cytomimetic applications. Chem Soc Rev 2012(41):5986-5997. https://doi.org/10.1039/C2CS35130G

4. Brenner S, Horne RW (1959) A negative staining method for high resolution electron microscopy of viruses. Biochim Biophys Acta 34:103-110. https://doi.org/10.1016/0006-3002(59)90237-9

5. De Carlo S, Harris JR (2011) Negative staining and cryo-negative staining of macromolecules and viruses for TEM. Micron 2011(42): 117-131. https://doi.org/10.1016/j.micron.2010.06.003 
6. Scarff CA, Fuller MJG, Thompson RF, Iadaza MG (2018) Variations on negative stain electron microscopy methods: tools for tackling challenging systems. J Vis Exp 2018(132):57199. https://doi.org/10.3791/57199

7. Dubochet J, McDowall AW (1981) Vitrification of pure water for electron microscopy. J Microsc 124:3-4. https://doi.org/10.1111/j. 1365-2818.1981.tb02483.x

8. Cheng Y, Grigorieff N, Penczek PA, Walz T (2015) A primer to single-particle cryo-electron microscopy. Cell 161:438-449. https:// doi.org/10.1016/j.cell.2015.03.050

9. Nogales E, Scheres SH (2015) Cryo-EM: a unique tool for the visualization of macromolecular complexity. Mol Cell 58:677689. https://doi.org/10.1016/j.molcel.2015.02.019

10. Sigworth FJ (2016) Principles of cryo-EM single-particle image processing. Microscopy-Jpn 65(1):57-67. https://doi.org/10.1093/ jmicro/dfv370

11. Radermacher M, Wagenknecht T, Verschoor A, Frank J (1987) Three-dimensional reconstruction from a single-exposure, random conical tilt series applied to the 50S ribosomal subunit of Escherichia coli. J Microsc 146:113-136. https://doi.org/10.1111/ j.1365-2818.1987.tb01333.x

12. Van Heel M (1987) Angular reconstitution: a posteriori assignment of projection directions for 3D reconstruction. Ultramicroscopy. 21(2):111-123. https://doi.org/10.1016/0304-3991(87)90078-7

13. Egelman EH (2000) A robust algorithm for the reconstruction of helical filaments using single-particle methods. Ultramicroscopy 85:225-234. https://doi.org/10.1016/S0304-3991(00)00062-0

14. Kühlbrandt W (2014) Biochemistry. The resolution revolution. Science 343:1443-1444. https://doi.org/10.1126/science.1251652

15. McMullan G, Faruqi AR, Henderson R (2016) Direct electron detectors. Methods Enzymol 579:1-17. https://doi.org/10.1016/bs. mie.2016.05.056

16. Brilot AF, Chen JZ, Cheng A, Pan J, Harrison SC, Potter CS, Carragher B, Henderson R, Grigorieff N (2012) Beam-induced motion of vitrified specimen on holey carbon film. J Struct Biol 177: 630-637. https://doi.org/10.1016/j.jsb.2012.02.003

17. Scheres SH (2012) RELION: implementation of a Bayesian approach to cryo-EM structure determination. J Struct Biol 180: 519-530. https://doi.org/10.1016/j.jsb.2012.09.006

18. Danev R, Buijsse B, Khoshouei M, Plitzko JM, Baumeister W (2014) Volta potential phase plate for in-focus phase contrast transmission electron microscopy. Proc Natl Acad Sci U S A 111: 15635-15640. https://doi.org/10.1073/pnas.1418377111

19. Danev R, Nagayama K (2008) Single particle analysis based on Zernike phase contrast transmission electron microscopy. J Struct Biol 161(2):211-218. https://doi.org/10.1016/j.jsb.2007.10.015

20. Danev R, Baumeister W (2017) Expanding the boundaries of cryoEM with phase plates. Curr Opin Struct Biol 46:87-94. https://doi. org/10.1016/j.sbi.2017.06.006

21. Ma K, Gong Y, Aubert T, Turker MZ, Kao T, Doerschuk PC, Wiesner U (2018) Self-assembly of highly symmetrical, ultrasmall inorganic cages directed by surfactant micelles. Nature 558:577580. https://doi.org/10.1038/s41586-018-0221-0

22. Vanhecke D, Asano S, Kochovski Z, Fernández-Busnadiego R, Schrod N, Baumeister W, Lučić V (2011) Cryo-electron tomography: methodology, developments and biological applications. J Microsc 242(3):221-227. https://doi.org/10.1111/j.1365-2818. 2010.03478.x

23. Ercius P, Alaidi O, Rames MJ, Ren G (2015) Electron tomography: a three-dimensional analytic tool for hard and soft materials research. Adv Mater 27(38):5638-5663. https://doi.org/10.1002/ adma.201501015

24. Nudelman F, de With G, Sommerdijk NAJM (2011) Cryo-electron tomography: 3-dimensional imaging of soft matter. Soft Matter 7(1):17-24. https://doi.org/10.1039/C0SM00441C
25. Lučić V, Foerster F, Baumeister W (2005) Structural studies by electron tomography: from cells to molecules. Annu Rev Biochem 74:833-865. https://doi.org/10.1146/annurev.biochem. 73.011303.074112

26. Mastronarde DN (1997) Dual-axis tomography: an approach with alignment methods that preserve resolution. J Struct Biol 120(3): 343-352. https://doi.org/10.1006/jsbi.1997.3919

27. Gilbert PF (1972) The reconstruction of a three-dimensional structure from projections and its application to electron microscopy. II Direct methods. Proc R Soc Lond B Biol Sci 182(1066):89-102. https://doi.org/10.1098/rspb.1972.0068

28. Gilbert P (1972) Iterative methods for the three-dimensional reconstruction of an object from projections. J Theor Biol 36(1):105-117. https://doi.org/10.1016/0022-5193(72)90180-4

29. Marabini R, Herman GT, Carazo JM (1998) 3D reconstruction in electron microscopy using ART with smooth spherically symmetric volume elements (blobs). Ultramicroscopy 72(1):53-65. https://doi. org/10.1016/S0304-3991(97)00127-7

30. Rigort A, Bauerlein FJ, Villa E, Eibauer M, Laugks T, Baumeister W, Plitzko JM (2012) Focused ion beam micromachining of eukaryotic cells for cryoelectron tomography. Proc Natl Acad Sci USA 109:4449-4454. https://doi.org/10.1073/pnas.1201333109

31. Lucic V, Fernández-Busnadiego R, Laugks U, Baumeister W (2016) Hierarchical detection and analysis of macromolecular complexes in cryo-electron tomograms using Pyto software. J Struct Biol 196:503-514. https://doi.org/10.1016/j.jsb.2016.10.004

32. Chen M, Dai W, Sun SY, Jonasch D, He CY, Schmid MF, Chiu W, Ludtke SJ (2017) Convolutional neural networks for automated annotation of cellular cryo-electron tomograms. Nat Methods 14: 983-985. https://doi.org/10.1038/nmeth.4405

33. Martinez-Sanchez A, Kochovski Z, Laugks U, zum Alten Borgloh JM, Chakraborty S, Pfeffer S, Baumeister W, Lučić V (2020, 2020) Template-free detection and classification of membrane-bound complexes in cryo-electron tomograms. Nat Methods. https://doi. org/10.1038/s41592-019-0675-5

34. Miao L, Duan H, Liu M, Lu W, Zhu D, Chen T (2017) Poly(ionic liquid)-derived, N, S-codoped ultramicroporous carbon nanoparticles for supercapacitors. Chem Eng J 317:651-659. https://doi.org/ 10.1016/j.cej.2017.02.110

35. Qian W, Texter J, Yan F (2017) Frontiers in poly(ionic liquid)s: syntheses and applications. Chem Soc Rev 46:1124-1159. https:// doi.org/10.1039/C6CS00620E

36. Herrmann S, De Matteis L, de la Fuente JM, Mitchell SG, Streb C (2017) Removal of multiple contaminants from water by polyoxometalate supported ionic liquid phases (POM-SILPs). Angew Chem Int Ed 56:1667-1670. https://doi.org/10.1002/anie. 201611072

37. Chen G, Hou W, Li J, Wang X, Zhou Y, Wang J (2016) Ionic selfassembly affords mesoporous ionic networks by crosslinking linear polyviologens with polyoxometalate clusters. Dalton Trans 45: 4504-4508. https://doi.org/10.1039/c6dt00070c

38. Zhang W, Kochovski Z, Lu Y, Schmidt BVKJ, Antonietti M, Yuan J (2016) Internal morphology-controllable self-assembly in poly(ionic liquid) nanoparticles. ACS Nano 10(8):7731-7737. https://doi.org/10.1021/acsnano.6b03135

39. Yang G, Zhang X, Kochovski Z, Zhang Y, Dai B, Sakai F, Jiang L, Lu Y, Ballauff M, Li X (2016) Precise and reversible proteinmicrotubule-like structure with helicity driven by dual Supramolecular interactions. J Am Chem Soc 2016(138):1932 1937. https://doi.org/10.1021/jacs.5b11733

40. Yang G, Ding HM, Kochovski Z, Hu RT, Lu Y, Ma YQ, Chen GS, Jiang M (2017) Highly ordered self-assembly of native proteins into $1 \mathrm{D}, 2 \mathrm{D}$, and 3D structures modulated by the tether length of assembly-inducing ligands. Angew Chem Int Ed 56:1069110695. https://doi.org/10.1002/anie.201703052 
Publisher's note Springer Nature remains neutral with regard to jurisdictional claims in published maps and institutional affiliations.

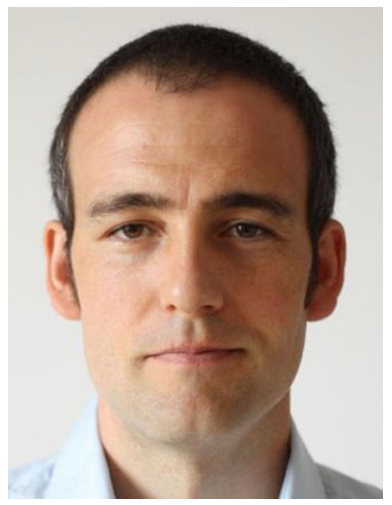

Zdravko Kochovski finished his undergraduate studies in 2006 at the Jacobs University Bremen (back then International University Bremen), obtaining a double BSc degree in B i o informatics and Computational Biology \& Electrical Engineering and Computer Science. He obtained his MSc in Computational Biology at the same university in 2008 and then moved to Munich for his $\mathrm{PhD}$ in Biophysics, working in the Molecular Structural Biology group of Prof. Wolfgang Baumeister at the Max-Planck Institute of Biochemistry. During his $\mathrm{PhD}$, he investigated the ultrastructure of individual synapses and isolated synaptic complexes by means of cryo-ET and cryo-EM SPA. In 2014, he moved to Berlin for a postdoc in the Institute of Soft Matter and Functional Materials at the HelmholtzZentrum Berlin für Materialien und Energie (HZB), headed by Prof. Matthias Ballauff. During this time, he has been responsible for running the Cryo-EM lab at the institute. Since 2019, he is a research scientist in the Institute for Electrochemical Energy Storage at HZB with a strong focus on cryo-EM. He enjoyed working with Matthias for over 5 years and thanks him for his support during that time.

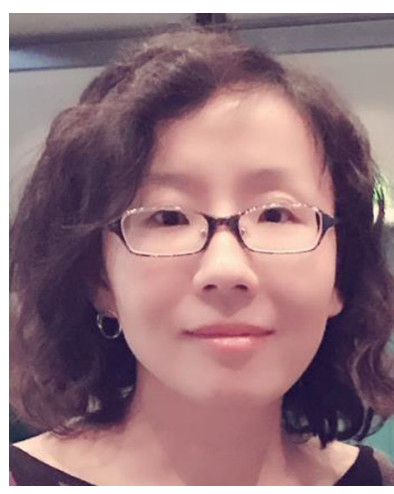

Guosong Chen studied chemistry at Nankai University, where she obtained her B.Sc. in 2001. In 2006, she received her Ph.D in the same university in supramolecular chemistry. After her postdoctoral studies in carbohydrate chemistry at Iowa State University, she moved to Fudan University in Dec. 2008, where she started as a lecturer in the Macromolecular Science Department. Then she was promoted to associate professor in 2011 , professor in 2014 .

Recently, her research focus has been reoriented to carbohydrate-based macromolecular self-assembly and its biological functions. She was elected as Fellow of Royal Chemical Society (FRSC) and now serves as Associate Editor of ACS Macro Letters and international board member for Polymer Chemistry, Bioconjugate Chemistry, Polymer International, etc. She was hosted by Prof. Matthias Ballauff with her Alexander von Humboldt Foundation for experienced researchers. She deeply thanks Matthias for the opportunity to work with him and his excellent colleagues as well as for all the mentor and support. Guosong hopes to visit him frequently when she has a chance to visit Berlin.

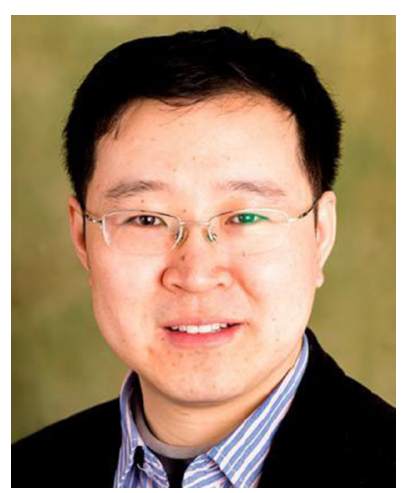

Jiayin Yuan studied chemistry at Shanghai Jiao Tong University (China) from 1998 to 2002 . He received a MSc degree from Uni. Siegen (Germany) in 2004 and PhD from Uni. Bayreuth (Germany) in 2009 with Axel H. E. Müller. In the Bayreuth period, he had joyful collaborations with Matthias Ballauff that ended up nicely with a Nature Materials paper (DOI: https://doi.org/10.1038/ nmat2232). Matthias Ballauff also served in his $\mathrm{PhD}$ defense committee. He joined the Max Planck Institute of Colloids and Interfaces in Potsdam first as a postdoctoral researcher (with Markus Antonietti) and then a research group leader. He received the prestigious European Research Council (ERC) Starting Grant in 2014, Dr. Hermann-Schnell Award in 2015, the Dozentenpreis from the Fund of Chemical Industry in 2016, and a Wallenberg Academy Fellowship from the Kunt and Alice Wallenberg Foundation in 2017 in Sweden. He completed his Habilitation in 2015 in Germany, and after an associate professor appointment at Clarkson University (USA, 2017), he is since Dec. 2018 a Full Professor at Stockholm University (Sweden).

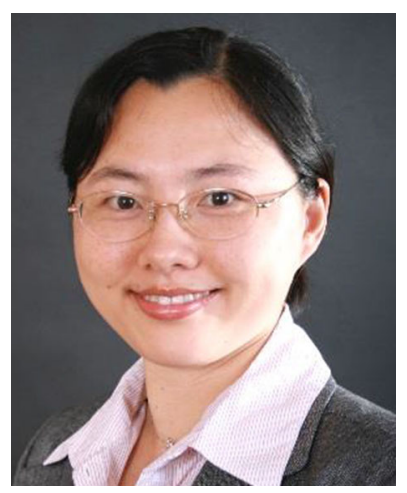

Yan Lu studied chemistry at Donghua University (China) in 1994. She received her MSc from Donghua University in 2001. In 2005, she received her $\mathrm{PhD}$ with summa cum laude under the supervision of Prof. H.-J. P. Adler in macromolecular chemistry at the Dresden University of Technology. After which, she worked first as postdoctor then research scientist in Prof. Matthias Ballauff's group at the University of Bayreuth. Since 2009, she joined the Helmholtz-Zentrum Berlin für Materialien und Energie (HZB) as a group leader in Colloid Chemistry in the Institute of Soft Matter and Functional Materials, where Matthias is the head of the institute. She received "Dr. Hermann-SchnellStipendium" by the German Chemical Society (GDCh) in 2011. She was selected as top female researchers (W2/W3 Programme) in Helmholtz Association in 2015. Since 2017, she is a professor in the Institute of Chemistry at the University of Potsdam. Since 2019, she is the acting head of the Institute for Electrochemical Energy Storage at HZB. She worked together with Matthias since 2005 for more than 14 years. She enjoyed so much the time she was working with Matthias and appreciated his support over the last years. 